\title{
Análisis de la campaña \#PrimAcoso: un continuo de violencias sexuales
}

\section{Analysis of the \#FirstSexualHarassment Campaign: a continuum of sexual violence}

\author{
Yolanda Rodríguez-Castro (D) https://orcid.org/0000-0001-9965-2348 \\ Universidad deVigo, España,yrcastro@uvigo.es
}

Rosana Martínez-Román (D) https://orcid.org/0000-0002-1122-5585

Universidad deVigo, España, rosana.mr@uvigo.es

Patricia Alonso-Ruido (D) https://orcid.org/0000-0002-5494-2795

Universidad de Santiago de Compostela, España, patricia.alonso.ruido@usc.es

María Victoria Carrera-Fernández (i) https://orcid.org/0000-0003-2158-3084

Universidad de Vigo, España, mavicarrera@uvigo.es

*Autora para

correspondencia:

Yolanda Rodríguez-Castro

yrcastro@uvigo.es

Recepción: 22/04/20

Aprobación: 04/07/20

Publicación: 28/01/21

\begin{abstract}
The aim of this study is to analyze the first experience of sexual violence that women have suffered through complaint tweets in the \#FirstSexualHarassment Campaign. A total of 2,887 tweets were posted with \#FirstSexualHarassment for one month, of which 688 were tweets and 2,199 were retweets. In addition, an analysis of the tweets posted confirmed that the $76 \%$ of the tweets were from cases of multiple violence suffered against female under the age of 18 (65\% were situations of sexual abuse of minors and $11 \%$ peer sexual harassment) and $24 \%$ to multiple violence against women adults $(21.3 \%$ sexual harassment, $1.6 \%$ harassment based on sex and $1.1 \%$ sexual assault). Therefore, sexual violence is a reality that women mostly suffer. Their first experience of violence in the childhood stage. Therefore, sex education is important to prevent sexual violence.
\end{abstract}

Key words: sexual violence, sexual abuse, sexual harassment, sexual assault.

Resumen: El objetivo de este estudio es analizar la primera experiencia de violencia sexual que han sufrido las mujeres a través de los tuits de denuncia en la Campaña \#PrimAcoso. Durante un mes se identificó un total de 2,887 tuits publicados con el hastag \#PrimerAcoso, de los cuales 688 eran tuits y 2,199 , retuits. Los resultados muestran que $76 \%$ de los tuits aluden a casos de múltiples violencias contra menores de 18 años (65\% fueron situaciones de abuso sexual a menores, y $11 \%$ acoso sexual entre iguales) y $24 \%$ a múltiples violencias contra mujeres adultas $(21.3 \%$ de acoso sexual, $1.6 \%$ de acoso por razón de sexo y $1.1 \%$ de agresión sexual). La violencia sexual es una realidad que sufren mayoritariamente las mujeres, y su primera experiencia se ubica en la infancia. Por ello es importante la educación sexual para prevenir ese problema.

Palabras clave: violencias sexuales, abuso sexual, acoso sexual, agresión sexual. 


\section{Introducción}

La violencia contra la mujer es una realidad que trasciende las fronteras físicas y adquiere magnitudes globales, convirtiéndose en la más generalizada y menos reconocida del mundo (Fitzgerald, 2017). Las experiencias de violencia sexual no son hechos aislados, sino un continuo de múltiples violencias que sufren las mujeres a lo largo de su vida (Kelly, 1988; Hayes y Dragiewicz, 2018).

Con base en los posicionamientos teóricos de Kelly (1988) dentro del continuum de las violencias sexuales que padecen las mujeres, se sitúa el inicio de aquellas en la infancia, las cuales se perpetúan durante toda la vida, como una vivencia continuada y en numerosas ocasiones invisibilizada. La Organización Mundial de la Salud (OMS, 2005: 161) define la violencia sexual como:

todo acto sexual, la tentativa de consumar un acto sexual, los comentarios o insinuaciones sexuales no deseados, o las acciones para comercializar o utilizar de cualquier otro modo la sexualidad de una persona mediante coacción por otra persona, independientemente de la relación de ésta con la víctima, en cualquier ámbito, incluidos el hogar y el lugar de trabajo.

Esta definición también incluye como violencia sexual a la coacción con una amplia gama de grados de uso de la fuerza física (desde un nivel leve y medio hasta grave), la agresión en los órganos sexuales y mutilación femenina, el acoso sexual, el acoso por razón de sexo, el matrimonio forzado, las inspecciones para comprobar la virginidad, la prostitución obligada y todos aquellos actos que afecten la integridad sexual de la mujer (OMS, 2005).

Para entender esta perspectiva de la violencia sexual contra las mujeres como una realidad inmutable y bajo el "pensamiento del continuum" para transformarse en una parte inevitable de la existencia de ser mujer (Tuerkheimer, 1997), es importante enmarcarla dentro de la ideología patriarcal que reproduce desigualdades de género en diferentes contextos (público y privado), de las relaciones (íntimas, familiares, escolares, conocidos, desconocidos), de cuestiones temporales (una vez, repetidas, continuadas) y culturales (Boyle, 2019). Al mismo tiempo que sigue perpetuando la masculinidad hegemónica, donde los hombres asumen el poder y someten a las mujeres mediante la utilización de sus cuerpos y de su sexualidad (Rodríguez et al., 2018).

De esta forma, los hombres tienen el privilegio de desplegar un amplio abanico de estrategias de coerción sexual contra las mujeres de cualquier edad, porque saben que "pueden hacerlo" al sentirse apoyados por las condiciones de ventaja que les otorga la ideología patriarcal (Lameiras et al., 2010). 


\section{El inicio de las violencias sexuales}

En la infancia y la adolescencia es cuando comienzan las primeras violencias sexuales (Dekeseredy et al., 2019), por ello centraremos el foco de atención principalmente en el abuso sexual a menores y el acoso sexual entre iguales.

Abuso sexual a menores

La OMS (2005) define el abuso sexual infantil como la participación de un/a niño/a en una actividad sexual que él o ella no comprende completamente y en la cual no puede dar su consentimiento. Los datos disponibles a nivel mundial muestran que el abuso sexual infantil es un tipo de violencia bastante frecuente, en especial en niñas, después de la violencia física y emocional (Assink et al., 2019).

Diversas investigaciones internacionales ofrecen una amplia horquilla de prevalencia: entre 12 y $46.7 \%$ de las niñas y niños han sufrido abuso sexual infantil (Allroggen et al., 2017; Morrison-Beedy y Grove, 2018). En el contexto español, algunos estudios marcan el tramo de prevalencia entre $13.9 \mathrm{y}$ 36.5\% (López et al., 2017; Pereda et al., 2018). Este tipo conductas, según algunos estudios, también son perpetradas durante la adolescencia, situando a esta etapa como el periodo más vulnerable a los abusos sexuales, sobre todo entre los 12 y 18 años (Chandraratne et al., 2018; Karayianni et al., 2017). Sin embargo, otras investigaciones apuntan que la edad en que iniciaron dichos abusos es a los siete años, mostrando una prevalencia de entre 10 y 22.5\% (Pereda et al., 2018; Marcos et al.2017).

Acoso sexual entre iguales

Otra de las violencias de tipo sexual a las que aludiremos es el acoso entre iguales, sufrido durante la preadolescencia y adolescencia. Según Vega-Gea et al. (2016), podemos entender el acoso sexual entre iguales como un comportamiento de naturaleza sexual no deseado que causa angustia e incomodidad en las víctimas, lo cual puede interferir en la vida normal del estudiantado en la escuela. Este tipo de acoso incluye "diferentes comportamientos como insultos, rumores, comentarios sexuales, miradas, gestos, intentos de contactos personales y ataques físicos" (Ortega et al., 2010: 248).

Los estudios a nivel internacional señalan las altas tasas de prevalencia de acoso sexual entre adolescentes, que oscilan entre 20 y $84 \%$ (Bucchianeri et al., 2013; Charmaraman et al., 2013), donde los niños/chicos muestran mayor participación como agresores que las niñas/chicas (Attar-Schwartz, 
2013). En esta misma línea, el trabajo de Kaltiala-Heino et al. (2018) evidencia que $36.9 \%$ de adolescentes sufrió acoso por orientación sexual entre iguales, $9.6 \%$ atención sexual no deseada y $3 \%$ coerción sexual. Las chicas son quienes más sufrieron estas tres formas de acoso sexual frente a los chicos.

Las investigaciones en España siguen la línea de las internacionales con tasas de prevalencia similares. Por ejemplo, el trabajo de Vega-Gea et al. (2016) muestra que el acoso de tipo sexual es una realidad que afecta entre 3.7 y $7.1 \%$ de las niñas y adolescentes españolas. Destaca también que en la mayoría de los casos se perpetúa principalmente a través de conductas de tipo no verbal y verbal.

Otros estudios se centraron en analizar el acoso por orientación sexual entre iguales, como el de Morales et al. (2016), al evidenciar cómo la masculinidad-instrumentalidad, independientemente del sexo biológico del agresor/a, se relaciona con la mayor participación como persona agresora, y la expresividad-feminidad con la menor implicación como persona agresora, en especial para las chicas.

\section{El transcurso del continuum de las violencias sexuales}

El continuum de la violencia sexual "perdura" en la etapa adulta de una mujer, transformándose en conductas de acoso sexual, acoso por razón de sexo y agresión sexual.

Acoso sexual y acoso por razón de sexo

El término acoso sexual (sexual harassment) fue acuñado en 1974 por un grupo de feministas durante un curso en la Universidad de Cornell (Estados Unidos). Dicho grupo analizó las experiencias de acoso sexual que tenían las mujeres en la esfera profesional por parte de los hombres (Pernas et al., 2000). Las primeras denuncias de estas situaciones se hicieron en Estados Unidos, y después en los países europeos. Esto permitió que el acoso sexual en el ámbito laboral entrara en la agenda política: pasó de ser un "problema" de naturaleza privada para convertirse en uno público (Cuenca-Piqueras, 2017; Rodríguez et al., 2019).

A pesar de la variabilidad conceptual del acoso sexual y acoso por razón de sexo, existe cierto consenso a nivel europeo -recogido en España en la Ley Orgánica 3/2007 de 22 de marzo para la igualdad efectiva de mujeres y hombres (Gobierno de España, 2007) - cuando se define el acoso sexual como cualquier comportamiento, verbal o físico, de naturaleza sexual que 
tenga el propósito o produzca el efecto de atentar contra la dignidad de una persona, en particular cuando se crea un entorno intimidatorio, degradante $\mathrm{u}$ ofensivo.

El acoso también se define por razón de sexo como cualquier comportamiento realizado en función del sexo de una persona, con propósito o efecto de atentar contra su dignidad y de crear un entorno intimidatorio, degradante u ofensivo (Gobierno de España, 2007: 12). De este modo, podemos señalar que el acoso sexual es un concepto fuertemente marcado por su naturaleza sexual; mientras que el acoso por razón de sexo incluye las discriminaciones que reciben las mujeres o las minorías sexuales que no conforman la norma de género, sin la necesidad de que interfieran comportamientos sexuales (Bosch et al., 2012).

En la investigación de Kearl (2018), en Estados Unidos, 65\% de las mujeres afirmaron que fueron víctimas de silbidos, pitazos, miradas lascivas y gestos de naturaleza sexual en las calles de Nueva York. Además, 57\% de las mujeres sufrieron acoso verbal mediante comentarios no deseados y $46 \%$ recibieron insultos sexistas.

En Europa, los datos siguen la misma línea. El informe de la Agencia de los Derechos Fundamentales de la Unión Europea (2014) indica que entre 83 y 102 millones de mujeres (45-55\% de las mujeres) de los 28 países de la Unión Europea han experimentado por lo menos una forma de acoso sexual y que muy pocas se atreven a denunciar. El reciente informe de la Fundación Jean Jaurés (Clavaud et al., 2018) evidencia que entre 49 y $61 \%$ de las mujeres revelan haber sufrido insultos sexistas o gestos groseros con connotación sexual por lo menos una vez a lo largo de su vida, y que más de $50 \%$ han sufrido acoso sexual alguna vez en su vida.

Agresión sexual

La agresión sexual constituye una de las formas más crueles de quebrantar los derechos humanos de las mujeres y produce consecuencias demoledoras en la calidad de vida. Dicha agresión no es un ataque aislado; durante toda su vida, las mujeres tienen una alta probabilidad de sufrirla (Igareda y Bodelón, 2014). La agresión sexual se define como cualquier acto que atenta contra la libertad sexual de otra persona, utilizando violencia o intimidación (Rodríguez et al., 2014). Incluye una serie de comportamientos que van desde tocamientos, conductas de sometimiento en la cual se obliga a una persona a desnudarse y actitudes indecentes, hasta penetraciones sin consentimiento. En definitiva, son actos que vulneran la libertad de la persona y que denigra, humilla y ofende a las mujeres y a las minorías sexuales (Rodríguez et al., 2014). 
La mayor parte del conocimiento sobre la prevalencia y el impacto de la agresión sexual se deriva de informes y estudios realizados en América del Norte y, cada vez más, en Europa occidental (Fedina et al., 2018; Schuster y Krahé, 2019). En Estados Unidos, en un informe nacional de 2015 sobre violencia sexual (Smith et al., 2018), se identificó que una de cada cinco mujeres sufrió violación o una tentativa de violación a lo largo de su vida; $21,3 \%$ afirmaron ser víctimas de una violación y $37 \%$ sufrieron contactos sexuales no deseados. En la misma línea, en un informe más reciente para la organización Stop Street Harassment (Kearl, 2018) 27\% de las mujeres afirmaron haber sufrido agresión sexual; y se situó la primera experiencia de este tipo entre los 14 y 17 años de edad.

A nivel europeo, el informe de la Fundación Jean Jaurés (Clavaud et al., 2018) evidencia que $48 \%$ de las mujeres europeas y $45 \%$ de las mujeres americanas han padecido algún tipo de agresión sexual como rozamientos, tocamientos sexuales no deseados o una agresión sexual con penetración a lo largo de su vida. En todos los casos, las menores de 25 años fueron el grupo más vulnerable. En esta dirección, según el estudio de metaanálisis de Dworkin et al. (2017), identifica que $28 \%$ de las mujeres sufrieron agresión sexual alguna vez en su vida y que $34 \%$ padecieron esta agresión mediante coacción.

En España, el reciente informe "Que se sepa. El macrocuestionario sobre violencias sexuales” (Cillán et al., 2020) recoge las experiencias de mujeres víctimas de violencias sexuales. Sus resultados evidencian que 53,14\% sufrieron una agresión sexual sin penetración; $17,75 \%$ afirmaron que se aprovecharon de ellas para mantener relaciones sexuales estando bajo los efectos del alcohol o de otras sustancias o en estado de inconsciencia; 41,64\% reportaron haber sufrido prácticas sexuales no consentidas y $15,74 \%$ dijeron que las forzaron a mantener relaciones sexuales. Sin embargo, sólo 3,12\% de las mujeres denunciaron estos episodios, lo cual supondría 2.675 denuncias por intento de violación.

Por lo tanto, en el último lustro, la violencia sexual ha adquirido una gran atención científica (Bows, 2018). Feministas como Cobo (2019) la sitúan en el epicentro de las demandas de la cuarta ola feminista, que está utilizando tanto el plano físico como el virtual. Así, la ubicuidad de Internet ha ofrecido a las víctimas un espacio de denuncia, a través del hashtag \#MeToo, para exponer lo que han sufrido a lo largo de sus vidas. Esta iniciativa, que fue puesta en marcha en las redes sociales por Alyssa Milano, se replicó rápidamente en más de 85 países, convirtiéndose en un movimiento internacional cuya finalidad es visibilizar las diversas violencias sexuales que han padecido las niñas 
y mujeres (Morrison-Beedy y Grove, 2018). Dichas herramientas virtuales han permitido a las víctimas expresarse y también tener contacto con otras víctimas para apoyarse y visibilizarse (Khatua et al., 2018).

En este contexto, el objetivo del presente estudio consistió en analizar los testimonios de mujeres sobre las violencias sexuales que han padecido, a través de la plataforma de Twitter con el hashtag \#PrimAcoso, que tiene un funcionamiento muy similar al del movimiento \#MeToo. En concreto, se animaba a las chicas y mujeres a que contaran su primera experiencia de acoso sexual.

\section{Metodología}

\section{Fuente informativa}

Desde la Concejalía de Igualdad del Ayuntamiento de Pontevedra, ubicada en el sudoeste de la comunidad autónoma de Galicia y el noroeste de España, se promovió una campaña -dentro de las actividades en contra de la violencia machista - a través de Twitter, en la que se pedía a las mujeres, a modo de denuncia: "comparte con nosotras/os la primera vez que te sentiste acosada por el hecho de ser mujer". Para ello se creó el hashtag \#PrimerAcoso, ${ }^{1}$ con el que durante un mes se identificó un total de 2.887 tuits, de los cuales 688 eran tuits y 2.199 eran retuits.

\section{Procedimiento y análisis de datos}

El análisis de los comentarios vinculados al hashtag \#PrimerAcoso fue gestionado a través de la metodología cualitativa, que permite "comprender, explicar, describir o predecir un fenómeno" (Izcara-Palacios, 2014: 17); en este caso, las vivencias del primer acoso sexual que sufrieron las mujeres, reflejándolas en las redes sociales.

Los tuits publicados en Twitter con el hashtag \#PrimerAcoso fueron descargados en una tabla de Excel con la aplicación de Twlets (Twitter to Excel). Posteriormente, los tuits se pasaron a un documento con extensión .rft para analizarlos con el programa de tratamiento y gestión de la información Atlas.Ti, v.8. El uso de este software permitió examinar la información a través de una clasificación por pares por parte de las investigadoras. Así, mediante el soporte Atlas.Ti se analizó el contenido de los tuits. La unidad

1 https://twitter.com/PrimeiroAcoso 
hermenéutica se examinó con un documento primario, 85 códigos, 1,440 citas, un memo y 10 familias. La codificación de los tuits se hizo expresando el sexo de la persona $(\mathrm{M}=$ mujer $)$ y el número de la cita del tuit.

Finalmente, del total de los tuits $(\mathrm{n}=2887)$, se seleccionaron 688 para estudiarlos, ya que los retuits solamente publicaban el tuit de otra persona y no aportaban ningún dato nuevo. Así, el análisis de los tuits se organizó en dos categorías principales. En la primera se recogieron las múltiples violencias hacia las niñas, y en la segunda se aglutinaron las múltiples violencias que sufren las mujeres adultas. A su vez, estas categorías se desglosaron en categorías secundarias y terciarias que se desarrollan en el apartado de resultados.

\section{Resultados}

El análisis de los 688 tuits de la campaña con el hastag \#PrimerAcoso permitió identificar el continuum de las múltiples violencias que sufren las niñas, las adolescentes y las mujeres a lo largo de su vida. El 76\% de los tuits aludían a casos contra menores de 18 años, y $24 \%$ a casos contra mujeres mayores de 18 años.

\section{Las múltiples violencias contra menores}

En esta categoría se identificaron 516 tuits, de los cuales 447 denunciaban situaciones de abusos y agresiones sexuales contra menores, y 69 relataban situaciones de acoso sexual entre iguales.

Abusos y agresiones sexuales a menores

Al examinar los tuits de las víctimas, se pudo identificar el tipo de abuso vivido, las características de quien lo sufrió, el lugar y las características del agresor.

a) Tipo de abuso

En relación con la tipología de abusos y agresiones sexuales durante la infancia/adolescencia relatados, identificamos abusos que se producen sin contacto físico y otros con contacto físico.

En cuanto a los casos sin contacto físico, las participantes padecieron amenazas directas o indirectas y comentarios sexuales no deseados sobre su apariencia física y su cuerpo sexuado, además de miradas desagradables y situaciones de exhibicionismo. Una cuarta parte dijo haber recibido abusos verbales con connotaciones sexuales. 
Consiguió mi teléfono y me decía que me iba a violar ( $\mathrm{M}, 1: 13)$.

Me amenazaba con un cuchillo (M, 1:502).

Por la calle me gritaba cosas (M, 1:22).

Nos enseña su pene en la calle (M, 1:126).

Respecto a los abusos sexuales con contacto físico, las mujeres relataron situaciones que experimentaron de niñas y adolescentes en las que su cuerpo se convirtió en un objeto de deseo para los agresores, quienes las tocaron en alguna parte de su cuerpo sin consentimiento.

Me tocó el culo (M, 1:67).

Me pellizcó los pechos y me dijo: "Ya estás hecha una mujer" (M, 1:109).

Me pasó la mano por el pubis y me decía: “Tendrás ya el pubis negro” (M, 1:959).

Me tocaba las piernas e intentaba meter sus dedos dentro de mis bragas (M, 1:449).

Me cogió en el coló y tuvo una erección. Me dejó el vestido empapado (M, 1:1027).

Cabe destacar que varias mujeres indicaron haber sido víctimas de "intentos de agresión sexual", señalando cómo fueron agarradas o empujadas de forma violenta por parte de los agresores o cómo intentaron introducirlas en un coche.

En una ocasión me querían obligar a tener sexo, me escapé (M, 1:398).

Volviendo a casa, un violador se me tiró encima y no me soltaba (M, 1:945).

Abrió la puerta e intentó meterme en su coche contra mi voluntad (M, 1:237).

b) Características de la víctima

Aquí surgen dos subcategorías relativas al género y la edad que tenían cuando sufrieron su primer abuso sexual.

En la totalidad de tuits que aludían a la situación de abuso sexual, aunque no se identificaba explícitamente el género de la persona, mediante el análisis de sus discursos pudimos encontrar que en todos los casos se trataba de mujeres.

Yo también fui una niña acosada ( $M, 1: 39$ ).

Siendo una niña de nueve o diez años sufrí abusos sexuales (M, 1:267).

En relación con la edad de la víctima, observamos que entre los cuatro y los 18 años sufrieron el abuso sexual; en 90\% de los casos las víctimas eran menores de 16 años. No obstante, el tramo de edad en el cual estas personas sufrieron más abusos se sitúa entre 8 y 12 años, siendo los 10 la edad más señalada. 
Con cuatro años (M, 1:934).

Creo que la primera vez debía tener entre cinco y siete años ( $M, 1: 126)$.

Siendo una niña de nueve o 10 años (M, 1:267).

Con 10 o 12 años (M, 1:289).

c) Lugar donde se perpetró el abuso sexual

Las mujeres contaron haber sufrido abusos sexuales cuando eran niñas tanto en espacios públicos como privados, aunque en estos últimos con mayor frecuencia. Un tercio de los casos ocurrió en un espacio público, como la calle, un camino o en una feria. Las mujeres también recuerdan que fueron atacadas en lugares próximos a su domicilio, e incluso en su portal o en las escaleras de su casa.

En la calle (M, 1:65).

Por la tarde, delante de mi casa (M, 1:602).

Se metió en el ascensor de mi edificio (M, 1:291).

Las víctimas señalaron que otros espacios públicos donde sufrieron la agresión fueron: centros educativos, instituciones eclesiásticas, campamentos, piscinas, cines e inclusive medios de transporte.

En el recreo del colegio (M, 1:62).

En la sacristía, preparando la primera comunión (M, 1:443).

En el campamento (M, 1:575).

En la piscina $(\mathrm{M}, 1: 321)$.

En el autobús del cole (M, 1:268).

Sin embargo, cabe destacar que la mayoría de los abusos sexuales se llevaron a cabo en espacios privados, como en su propia casa o en la casa de familiares o amistades.

En la casa de mi abuela (M, 1:388).

En mi casa (M, 1:166).

En la casa de mi amiga (M, 1:189).

d) Características del agresor

El análisis de los relatos permitió identificar el tipo de vinculación del agresor con la víctima, destacando así a personas conocidas y desconocidas. En su mayoría, los abusos sexuales fueron perpetrados por personas conocidas por 
la víctima. Resalta que en uno de cada ocho casos el abusador era un familiar directo de la víctima: su padre, abuelo o tío.

Un amigo de mis padres (M, 1:33).

El señor del bar de los bocadillos (M, 1:827).

Un vecino (M, 1:227).

Un familiar (M, 1:927).

Mi abuelo (M, 1:928).

En cambio, otras mujeres señalaron que sus agresores fueron miembros de la comunidad educativa, principalmente profesores, monitores y conserjes; así como miembros eclesiásticos de diversas congregaciones: sacerdotes y monjes.

A las chicas, el profesor nos hacía ir a corregir a su mesa, y mientras aprovechaba para tocarnos (M, 1:511).

Mi profesor de Filosofía nos restregaba su pene (M, 1: 675).

El conserje (M, 1:681).

El cura empezaba haciéndote cosquillas y luego ya pasaba a tocarte todo (M, 1:830).

En cuanto a los abusos perpetrados por personas sin vinculación con la víctima, las participantes indicaron que el agresor fue un hombre desconocido y adulto. También cabe destacar que en varios tuits las víctimas relataron que los abusos sexuales recibidos fueron cometidos por varios hombres a la vez.

Era un hombre que no conocía (M, 1:808).

En un Ford Fiesta color beis, un hombre mayor (M, 1:236).

Seis hombres adultos ( $M, 1: 788)$.

Un grupo de chicos (M, 1:112).

Acoso sexual entre iguales

Dentro de las múltiples violencias hacia los y las menores, el análisis evidenció casos de acoso sexual entre iguales, con $11 \%$ del total de las situaciones denunciadas en los tuits.

a) Tipo de acoso sexual entre iguales

En este apartado identificamos los acosos que se producen sin contacto físico y con contacto físico. Los primeros, a pesar de ser la minoría, aluden a las 
situaciones relacionadas con comentarios de tipo sexual ofensivos, referidos principalmente a la valoración del cuerpo de las chicas.

No me dejaba sola, "rubia, qué buena estás" (M, 1:398).

Me explicaba qué significaba follar y me decía que podíamos intentarlo (M, 1:648).

La mayoría de los casos de acoso sexual entre iguales hacen referencia a comportamientos que implican contacto físico. Nuevamente, el cuerpo de las niñas y de las chicas se convierte en un objeto de deseo de sus iguales por medio de tocamientos no deseados, como levantarles la falda para ver su ropa interior y rozarse contra sus cuerpos, empleando la violencia y ocasionando a veces daño físico.

Me levantaba la falda y me tocaba el culo (M, 1:550).

Te agarran y te inmovilizan sobre las mesas (M, 1:801).

En ese momento me cogió y me pellizcó el pubis con fuerza (M, 1:413).

Cabe señalar que en un tercio de los casos de acoso sexual entre iguales, las mujeres expresaron que cuando eran niñas sufrieron tocamientos no deseados y no consentidos por varios chicos a la vez. Los agresores actuaban en "manada" y empleaban en ocasiones la estrategia del juego para intimidar y abusar de la víctima.

Me rodeaban para tocarme ( $\mathrm{M}, 1: 387)$.

Jugaban a tocarnos el culo a las chicas en el recreo y a levantarnos las faldas (M,1:407).

Dos niños se dedicaban a cazar niñas y llevarlas a su cueva (al soportal del patio) y allí retenidas, te tocaban $(\mathrm{M}, 1: 839)$.

b) Características de la víctima

Mediante el análisis de contenido, identificamos que en la mayoría de los tuits las niñas son las principales víctimas.

c) Lugar donde se produce el acoso sexual entre iguales

Los relatos revelan mayoritariamente situaciones en el contexto escolar como en el aula, durante los recreos o en el transporte escolar, pero también en el contexto no escolar asociado a campamentos o clases extraescolares.

En el colegio (M, 1:530).

Bus escolar (M, 1:796).

Campamento de la Xunta de Galicia (M, 1:396).

Clases de judo (M, 1:463). 
d) Características del abusador

El análisis de los tuits de las víctimas permitió identificar el género, la edad del perpetrador y el tipo de vínculo. De forma general, podemos señalar que en todos los casos el agresor era un chico. La mayoría de los agresores tenía una edad similar a la de la víctima; solamente en cuatro casos el agresor era un chico mayor. Y los agresores eran conocidos de la víctima.

Chico de secundaria (M, 1:470).

Un niño de mi clase (M, 1:549).

Mis compañeros (M, 1:868).

\section{Las múltiples violencias hacia las mujeres adultas}

Esta segunda categoría principal representa $24 \%$ de las situaciones denunciadas a través de los tuits $(\mathrm{n}=161)$, por lo que se identificaron tres tipos de violencias: 1) acoso sexual, 2) acoso por razón de sexo, y 3) agresión sexual.

\section{Acoso sexual}

Cabe destacar que 143 tuits (21.3\%) publicados en la campaña \#PrimAcoso eran relativos a situaciones de acoso sexual que vivieron las mujeres. En el análisis se identificó el tipo de abuso, las características de la víctima, el lugar y las características del acosador.

\section{a) Tipo de acoso sexual}

Las conductas se clasificaron en aquellas sin contacto físico y con contacto físico. En las primeras, a pesar de ser las menos relatadas por las víctimas, encontramos que fueron fundamentalmente conductas verbales, como, por ejemplo, piropos.

Me dijo groserías por la calle (M, 1:774).

Me pidió la hora y a continuación que le diera un beso (M, 1:141).

Respecto a las situaciones que implican contacto físico, se halló que son el tipo de acoso sexual más frecuente, sufrido por la mayoría de las víctimas a través de tocamientos sin su consentimiento.

Me tocó el culo (M, 1:505).

Comenzó a tocarme los pechos (M, 1:276).

Vino por detrás y me tocó entre las piernas (M, 1:881).

Aprovechó para abalanzarse sobre mí y darme un abrazo (M, 1:371). 
Asimismo, diversas víctimas indicaron que estos comportamientos son tan frecuentes en su vida cotidiana que incluso ya están normalizados socialmente, lo cual provoca que se vean como "juegos" o "bromas".

No podías ir a la pista de baile de arriba porque te tocaban entera y allí era "lo normal" (M, 1:524).

b) Características de la víctima

Todos los casos relatados eran de mujeres que sufrieron este tipo de acoso, principalmente entre los 18 y 20 años.

Cuando tenía 18/19 años (M, 1:535).

Mi primer \#PrimAcoso fue a los 20 años (M, 1:878).

c) Lugar donde se produjo el acoso

Tres cuartas partes de las participantes manifiestaron haber sufrido acoso sexual, sobre todo en espacios públicos, y en menor medida en espacios privados.

De las primeras veces que fui sola al cine (M, 1:80).

En la calle (M, 1:407).

En la primera entrevista de trabajo (M, 1:349).

En mi casa (M, 1:1020).

d) Características del acosador

Mediante el análisis se demostró que no existe un perfil definido de la persona acosadora, ya que las víctimas sufrieron este tipo de conductas por parte de conocidos y desconocidos. Más de la mitad de las situaciones de acoso sexual relatadas en los tuits fue perpetrada por personas "desconocidas" para la víctima. En algunos casos, incluso, las mujeres narraron que fueron acosadas sexualmente por varios hombres al mismo tiempo.

Un obrero (M, 1:307).

Un tipo de 30 años y pico ( $M, 1: 686)$.

El técnico de gas (M, 1:5).

Por siete hombres (M, 1:773,775).

En cuanto a las situaciones de acoso con personas conocidas, las víctimas señalaron a miembros de su familia, amigos, vecinos, superiores e incluso profesores. 
Mi "amigo" de siempre (M, 1:314).

Monitor de autoescola (M, 1:552).

Tíos, amigos, primos, vecinos, profesores, curas (M, 1:688-689).

Acoso por razón de sexo

Se identificaron 11 tuits (1.6\%) que permitieron establecer la tipología del acoso, las características de la víctima, el lugar y las características del acosador de este tipo de violencia.

\section{a) Tipo de acoso}

Los relatos de las víctimas muestran que en todos los casos las situaciones abarcaron tanto acoso verbal como no verbal. Respecto al primero, al que alude la mayoría de las víctimas, se trata fundamentalmente de comentarios ofensivos y vejatorios como piropos, seguidos de comentarios sexistas.

Todo tipo de bocinas, salvajadas e improperios desde coches hasta camiones (M, 1:615).

Cuando te gritan en la calle (M, 1:581).

Siempre había burlas sobre el físico (M, 1:844).

Le dijo a mi padre que estudiar a una hija era tirar con el dinero, porque mi futuro era casarme y parir (M, 1:76).

En relación con el acoso a través de conductas no verbales, las víctimas indicaron comportamientos discriminatorios sufridos a lo largo de su vida por el simple hecho de ser mujeres.

Testimonios cómo una persona era rechazaba para dar clases por ser mujer (M, 1:227).

Es acoso cobrar menos que un hombre por el mismo trabajo (M, 1:1018).

b) Características de la víctima

Todos los tuits examinados eran de mujeres que relataban las situaciones de acoso por razón sexual que habían vivido.

Cuando eres jefa, a los trabajadores no les gusta (M, 1:486).

c) Lugar donde se produjo el acoso

Las mayoría de las víctimas señalaron que fue en el espacio público, tanto en el ámbito laboral y académico como en la vía pública. 
Al comenzar en la universidad (M, 1:74).

En la calle (M, 1:581).

En la banda de gaitas que había en mi pueblo (M, 1:139).

d) Características del acosador

Las víctimas no proporcionaron demasiada información en cuanto al sexo de los acosadores. No obstante, observamos que pueden ser hombres conocidos aunque no cercanos.

Un tipo (M, 1:104).

Un compañero ( $M, 1: 90)$.

\section{Agresión sexual}

Mediante su relato en los tuits, $1.1 \%$ de las mujeres dijeron haber sido víctimas de una agresión sexual a lo largo de su vida o de intentos de agresión sexual. En todos los casos, eran mujeres jóvenes en el momento de la agresión, siendo el agresor un desconocido para la víctima, aunque en alguna situación era un amigo suyo.

Con 18 años, en una verbena, no lo conocía. Me cogió desprevenida y no me dejó mover. Y me hizo daño (M, 1:803-806).

A los 23 años un intento de violación por un amigo que me acompañaba a casa (M, 1:225).

\section{Discusión}

Con base en el objetivo de este estudio, se comprobó cómo las mujeres, mediante las nuevas tecnologías y en este caso sirviéndose de la red social de Twitter, han dado un paso hacia adelante para visibilizar y denunciar la violencia sexual que han padecido desde pequeñas. De hecho, $76 \%$ de los tuits aludían a situaciones sufridas cuando aún eran menores; de dicho porcentaje, $65 \%$ fueron de abusos a menores y $11 \%$ de acoso sexual entre iguales.

Respecto al tipo de abuso sexual, se encontró tanto con contacto físico como sin él. Las víctimas relataron mayoritariamente tocamientos y rozamientos no deseados en alguna parte sexuada de su cuerpo, incluso mediante el uso de la fuerza. Estos resultados se hallan en la línea de estudio de Chandraratne et al. (2018). Asimismo, más de una cuarta parte de los 
tuits aludía a abusos verbales con connotaciones sexuales, como en el estudio de Witt et al. (2018), donde se muestran prevalencias elevadas en el abuso sexual sin contacto físico.

En cuanto a la vinculación de la víctima con el agresor, la mayor parte de los abusos sexuales fueron perpetrados por personas conocidas de la víctima; incluso, en uno de cada ocho casos, el abusador fue un familiar directo. Estudios como el de González-García y Carrasco (2016) o el de Reed et al. (2019) señalan como agresores de menores sobre todo a familiares directos, mientras que investigaciones como la de Bogen et al. (2019) apuntan como principales agresores a profesores, vecinos o curas. Sólo un tercio de los tuits indicaba que los agresores eran desconocidos.

Este hecho está relacionado con que dos tercios de los casos se produjeron en el espacio privado: en la casa o en la de familiares y amistades. Solamente una tercera parte de las situaciones denunciadas tuvo lugar en espacios públicos pero próximos a su domicilio, como en el portal, en la escalera o en sitios relacionados con el centro educativo o la iglesia, o con el ocio y el tiempo libre en campamentos, piscinas o cines.

Otra de las violencias sexuales denunciadas en los tuits cuando aún eran chicas menores de edad se refería a situaciones de acoso sexual por parte de sus iguales (11\%). La mayoría de éstas, perpetradas por chicos, fueron con contacto físico, por ejemplo, al levantarles la falda para verles la ropa interior, rozarse contra su cuerpo, tocamientos en partes sexuales, etcétera.

De esta forma, los chicos tienden a objetivizar/cosificar el cuerpo de las chicas a través de un contacto físico no deseado y no permitido. Este tipo de acoso sexual es consentido por la sociedad patriarcal y no frena a los acosadores, pues ellos lo aprovechan para reforzar el "poder" que ejercen sobre la dimensión sexual de las chicas/mujeres (Rodríguez et al., 2019). Incluso llegan a considerar estas conductas como una diversión inofensiva o como una normalidad en las interacciones que marca el género (Quinn, 2002). Un tercio de los tuits sobre acoso sexual entre iguales señalaba que los agresores actuaban en grupo, emulando el "efecto manada" (Liang y Hu, 2018).

El 24\% de los tuits totales aludía a múltiples violencias sexuales sufridas por las mujeres en su vida adulta a través del acoso sexual, acoso por razón de sexo y agresión sexual. La primera es la situación más señalada con $21.3 \%$ de los tuits, y su materialización se lleva a cabo mayoritariamente por medio del contacto físico, como tocamientos y manoseos sin consentimiento, en especial en alguna parte sexuada de su cuerpo.

No obstante, las mujeres también reciben, aunque en menor medida, comentarios sexuales sobre sus atributos físicos, piropos, miradas incómodas e 
incluso persecución e insistencia para mantener relaciones sexuales. Ante las situaciones de acoso verbal, tal y como se evidencia en el estudio de Rodríguez-Martínez y Cuenca-Piqueras (2019), las mujeres no suelen identificar estos comportamientos como acoso sexual a pesar de sentirse incómodas, porque lo tienen normalizado en su vida. Sólo lo identifican cuando va acompañado de un tocamiento no deseado. Este hecho provoca que el acoso verbal esté menos visibilizado y denunciado.

En relación con la víctima del acoso sexual, las mujeres señalaron que sufrieron esas situaciones sobre todo cuando tenían entre 18 y 20 años. Asimismo, el acoso tuvo lugar fundamentalmente en espacios públicos, en particular en calles y lugares de ocio, pero también en centros educativos como la universidad. Así lo ha destacado el estudio de Kearl (2018), quien evidencia que el acoso sexual en la calle es un grave problema social, y, en menor medida, en espacios privados.

En cuanto al perfil del acosador, la mayoría son hombres desconocidos (Logan et al., 2015), y con menor frecuencia hombres conocidos por las víctimas, pertenecientes a su ámbito de amistades, laboral o académico. Esta realidad provoca en las mujeres una sensación de indefensión, por la frecuencia con la cual se producen este tipo de situaciones (Graham et al., 2014), y por la condescendencia social con la que se juzga el acoso sexual en espacios donde está culturalmente normalizado (Kavanaugh, 2014). Cabe señalar que en los espacios públicos, a menudo las mujeres se pueden sentir incómodas, inseguras y sexualmente intimidadas, sobre todo durante la noche (Kavanaugh, 2014).

Mediante los tuits analizados, se puso de manifiesto que junto con el acoso sexual, las mujeres también sufrieron acoso por razón de sexo. Éste representa $1.6 \%$ del total de las situaciones de violencia vividas por las mujeres y se materializa a través de diferentes conductas, incluyendo mayoritariamente el acoso verbal, por medio de comentarios sexistas que objetivizan a las mujeres y resaltan cualidades tradicionalmente asociadas a la expresividad-feminidad como la belleza o que castigan la transgresión de los estereotipos femeninos con insultos o juzgando su capacidad.

Según los tuits, estas situaciones ocurrieron en el espacio público, en concreto en el ámbito laboral y académico, pero también en las calles, y fueron perpetrados por hombres conocidos de la víctima, aunque no muy cercanos. El acoso por razón de sexo ha sido recientemente visibilizado en estudios con la comunidad universitaria (Lameiras et al., 2018), en la cual las estudiantes y las docentes manifestaron abiertamente que recibían algunos comentarios sexistas por parte de compañeros y profesores. 
Por último, nuestros resultados evidencian que $1.1 \%$ del total de los tuits son de mujeres que denunciaron haber sido víctimas de una agresión sexual a lo largo de su vida. Dos de ellas relataron dos intentos de violación que, afortunadamente, se frustraron porque pudieron escapar, y fueron perpetrados, en uno de los casos por un hombre desconocido, y en el otro, por un amigo de la víctima que la acompañaba a su casa supuestamente para protegerla.

En esta misma línea, estudios como el de Kearl (2018) sostienen que las agresiones sexuales son más frecuentes por parte de personas conocidas que por desconocidas.

\section{Conclusión}

Los resultados de este estudio visibilizan el carácter generalizado y normalizado de las violencias sexuales en la vida de las mujeres. Es más, se puede afirmar que la infancia es el periodo donde más de ellas recuerdan su primer episodio de violencia, en especial a través de abusos sexuales a menores. El acoso sexual y el acoso por razón de sexo, tanto en adolescentes como en mujeres adultas, en este orden, son otras de las variadas violencias relatadas por las participantes. Asimismo, el presente estudio reconoce la importancia de la narrativa de las víctimas como componente central en los movimientos sociales de las mujeres, ya que visibilizar sus voces y analizar sus experiencias es esencial para las investigaciones feministas que han permitido a las mujeres identificar y comprender la naturaleza estructural y de género en sus continuadas experiencias de violencia sexual a lo largo de su vida (Boyle, 2019).

Actualmente, el activismo feminista a través de las redes sociales está aumentando una conciencia igualitaria en la sociedad (Mendes et al., 2018). Así, movimientos como \#MeToo, \#TimeUp, \#TalkAboutIt, \#YoSiTeCreo (\#IBelieveYou), entre otros, han conseguido poner el foco de atención sobre esta realidad de violencias que padecen muchas mujeres y niñas, y que hasta ese momento estaban silenciadas (Park, 2017). Esto ha marcado un punto de inflexión al ayudar a las víctimas a romper el estigma social que hay sobre este tipo de violencias y a mostrar a la luz pública una realidad invisibilizada.

Por lo tanto, estas campañas han contribuido a un "pequeño" avance social en la lucha contra la violencia sexual en niñas y mujeres (Patrick, 2018), pero aún queda mucho camino. Consideramos necesario complementar estas iniciativas con una formación en educación sexual, implementando en el sistema escolar programas de dicha educación y prevención de la violencia sexual, destinados no sólo al alumnado, sino también a toda la comunidad 
educativa, incluyendo a las familias, con la finalidad de (re)construir una sociedad más justa e igualitaria, en la que mujeres y niñas puedan vivir libres de las violencias sexuales y de género.

\section{Referencias}

Agencia de los Derechos Fundamentales de la Unión Europea (2014), Violencia de género contra las mujeres: una encuesta a escala de la UE. Disponible en: https://fra.europa. eu/sites/default/files/fra-2014-vaw-survey-at-a-glance-oct14_es.pdf [5 de abril de 2019].

Allroggen, Marc et al. (2017), "Lifetime prevalence and incidence of sexual victimization of adolescents in institutional care", en Child Abuse y Neglect, vol. 66. DOI: 10.1016/j. chiabu.2017.02.015. Disponible en: https://www.sciencedirect.com/science/article/ pii/S0145213417300558?via\%3Dihub [6 de junio de 2019].

Assink, Mark et al. (2019), "Factores de riesgo para la victimización por abuso sexual infantil: una revisión metaanalítica”, en Boletín Psicológico, vol. 145, núm. 5. DOI: 10.1037/ bul0000188. Disponible en:

https://psycnet.apa.org/doiLanding?doi=10.1037\%2Fbul0000188 [13 de mayo de 2019].

Attar-Schwartz, Shalhevet (2013), "Runaway behavior among adolescents in residential care: The role of personal characteristics, victimization experiences while in care, social climate, and institutional factors", en Children and Youth Services Review, vol. 35, núm. 2. DOI: 10.1016/j.childyouth.2012.11.005. Disponible en:

https://www.sciencedirect.com/science/article/pii/S0190740912004082 [6 de junio de 2019].

Bogen, Katherine et al. (2019), "\#MeToo: Disclosure and Response to Sexual Victimization on Twitter", en Journal of Interpersonal Violence, vol. 1. Disponible en:

https://journals.sagepub.com/doi/pdf/10.1177/0886260519851211 [3 de junio de 2019].

Bosch, Esperanza et al. (2012), "El acoso sexual en el ámbito universitario. Elementos para mejorar la implementación de medidas de prevención, detección e intervención", en Estudios e Investigaciones, España: Ministerio de Sanidad, Servicios Sociales e Igualdad. Disponible en: http://www.inmujer.gob.es/eu/areasTematicas/estudios/ estudioslinea2014/docs/El_acoso_sexual_ambito_universitario.pdf $[19$ de abril de 2019].

Bows, Hannah (2018), "Sexual violence against older people: A review of the empirical literature”, en Trauma, Violence, \& Abuse, vol. 19, núm. 5. Disponible en: https://journals.sagepub.com/doi/pdf/10.1177/1524838016683455 [5 de abril de 2019].

Boyle, Karen (2019), "What's in a name? Theorising the inter-relationships of gender and violence", en Feminist Theory, vol. 20, núm.1. Disponible en: https://journals.sagepub. com/doi/pdf/10.1177/1464700118754957 [5 de abril de 2019].

Bucchianeri, Michaela et al. (2013), "Weightism, Racism, Classism, and Sexism: Shared Forms of Harassment in Adolescents", en Journal of Adolescent Health, vol. 53, núm. 1. DOI: 10.1016/j.jadohealth.2013.01.006 Disponible en: https://www.sciencedirect. com/science/article/pii/S1054139X13000475 [6 de junio de 2019]. 
Chandraratne, Nadeeka et al. (2018), "Physical, sexual and emotional abuse during childhood: experiences of a sample of Sri Lankan young adults", en Child abuse y neglect, vol. 81. DOI: 10.1016/j.chiabu.2018.05.004. Disponible en: https://www. sciencedirect.com/science/article/pii/S014521341830200X\#! [13 de mayo de 2019].

Charmaraman, Linda et al. (2013), "Is it bullying or sexual harassment? Knowledge, attitudes, and professional development experiences of middle school staff”, en Journal of school health, vol. 83, núm.6. Disponible en: https://onlinelibrary.wiley.com/doi/ full/10.1111/josh.12048 [6 de abril de 2019].

Cillán, Marta et al. (2020), "Que se sepa. El macrocuestionario sobre violencias sexuales". Disponible en: https://www.quesesepa.org/ [8 de julio de 2020].

Clavaud, Amandine et al. (2018), "Les femmes face aux violences sexuelles et le harcèlement dans la rue”. Disponible en: https://jean-jaures.org/sites/default/files/redac/commun/ productions/2018/enquete_harcelement.pdf [3 de junio de 2019].

Cobo, Rosa (2019), "La cuarta ola feminista y la violencia sexual", en Revista Universitaria de Cultura, núm. 22. Disponible en: https://riuma.uma.es/xmlui/bitstream/ handle/10630/17716/134\%20Cobo.pdf [6 de julio de 2020].

Cuenca Piqueras, Cristina (2017), El acoso sexual: un aspecto olvidado de la violencia de género, España: Centro de Investigaciones Sociológicas.

Dekeseredy, Walter et al. (2019), "Polyvictimization and the continuum of sexual abuse at a college campus: does negative peer support increase the likelihood of multiple victimizations?" en The British Journal of Criminology, vol. 59, núm. 2. DOI: 10.1093/bjc/azy036. Disponible en: https://academic.oup.com/bjc/ article/59/2/276/5085264 [8 de julio de 2020].

Dworkin, Emily et al. (2017), "Sexual assault victimization and psychopathology: A review and meta-analysis", en Clinical psychology review, vol. 56. DOI: 10.1016/j. cpr.2017.06.002. Disponible en:

https://www.sciencedirect.com/science/article/pii/S0272735817300880 [9 de mayo de 2019].

Fedina, Lisa et al. (2018), "Campus sexual assault: A Systematic Review of Prevalence Research from 2000 to 2015”, en Trauma, Violence \& Abuse, vol. 19, núm. 1. Disponible en: https://journals.sagepub.com/doi/full/10.1177/1524838016631129 [9 de mayo de 2019].

Fitzgerald, Louise (2017), "Still the last great open secret: Sexual harassment as systemic trauma”, en Journal of Trauma \& Dissociation, vol. 18, núm. 4. DOI:10.1080/152997 32.2017.1309143 Disponible en: https://www.tandfonline.com/doi/pdf/10.1080/1 5299732.2017.1309143?need Access=true [8 de julio de 2020].

González-García, Francisco y Carrasco, Miguel Ángel (2016), "Evaluación del perfil psicosocial en menores víctimas de abuso sexual: diferencias por sexo y edad”, en Revista de Psicología Clínica con niños y adolescentes, vol. 3, núm. 2. Disponible en: https:// dialnet.unirioja.es/servlet/articulo?codigo=5590678 [13 de mayo de 2019].

Graham, Kathryn et al. (2014), "Young women's risk of sexual aggression in bars: The roles of intoxication and peer social status”, en Drug y Alcohol Review, vol. 33, núm. 4. Disponible en: https://onlinelibrary.wiley.com/doi/full/10.1111/dar.12153 [10 de junio de 2019].

Hayes, Rebecca y Dragiewicz, Molly (2018), "Unsolicited dick pics: Erotica, exhibitionism or entitlement?", en Women's Studies International Forum, vol. 71. DOI: 10.1016/j. 
Convergencia Revista de Ciencias Sociales, vol. 28, 2021, Universidad Autónoma del Estado de México

wsif.2018.07.001. Disponible en: https://www.sciencedirect.com/science/article/pii/ S0277539518300384 [3 de junio de 2019].

Igareda, Noelia y Bodelón, Encarna (2014), "Las violencias sexuales en las universidades: cuando lo que no se denuncia no existe", en Revista Española de Investigación Criminológica, núm. 12. Disponible en: https://dialnet.unirioja.es/servlet/ articulo?codigo $=4783305$ [ 10 de junio de 2019].

Izcara-Palacios, Simón Pedro (2014), Manual de investigación cualitativa, México: Fontamara.

Kaltiala-Heino, Riittakerttu et al. (2018), "Experiences of sexual harassment are associated with the sexual behavior of 14-to 18-year-old adolescents", en Child abuse y neglect, vol. 77. DOI: 10.1016/j.chiabu.2017.12.014. Disponible en:

https://www.sciencedirect.com/science/article/pii/S0145213417304623 $\left[\begin{array}{ll}10 & \text { de }\end{array}\right.$ junio de 2019].

Karayianni, Eleni et al. (2017), "Prevalence, contexts, and correlates of child sexual abuse in Cyprus", en Child abuse y neglect, vol. 66. DOI: 10.1016/j.chiabu.2017.02.016. Disponible en: https://www.sciencedirect.com/science/article/pii/ S014521341730056X [13 de mayo de 2019].

Kavanaugh, Philip (2014), "Pathways on the sexual violence continuum: A lifestyles theory of victimization in urban nightlife", en Journal of Crime and Justice, vol. 38, núm. 4. DOI: 10.1080/0735648X.2014.904244. Disponible en: https://www.tandfonline. com/doi/abs/10.1080/0735648X.2014.904244 [5 de abril de 2019].

Khatua, Aparup et al. (2018), "Sonidos que rompen el silencio: Explorando la violencia sexual en twitter”, en IEEE / ACM International Conference on Advances in Social Networks Analysis and Mining (ASONAM). Disponible en: https://ieeexplore.ieee.org/ abstract $/$ document $/ 8508576$ ?casa_token=5Mq4yA8qxlEAAAAA:UENPBXoP_ VAnjll7wYi303sWyJB9DstNMRaZK-BJbQjt9wR1ttZzTotpBEv6eJfD0_qIW29o [6 de julio de 2020].

Kearl, Holly (2018), "The facts behind the \#Metoo movement: A National Study on Sexual Harassment and Assault. Stop Street Harassment”. Disponible en: http:// www.stopstreetharassment.org/wp-content/uploads/2018/01/Full-Report-2018National-Study-on-Sexual-Harassment-and-Assault.pdf [ 3 de junio de 2019].

Kelly, Liz (1988), Surviving Sexual Violence, Estados Unidos: University of Minnesota Press.

Lameiras, María et al. (2010), "La violencia sexual contra las mujeres: abordaje psicosocial", en Lameiras, María e Iglesias, Inés [eds.], Violencia de género: la violencia sexual a debate, España: Tirant lo Blanch.

Lameiras, María et al. (2018), Diagnose do acoso sexual e por razón de sexo na Universidade de Vigo, España: Universidad de Vigo.

Gobierno de España (2007), "Ley Orgánica 3/2007, de 22 de marzo, para la igualdad efectiva de mujeres y hombres", en Boletín Oficial del Estado, núm. 71. Disponible en: https://www.boe.es/buscar/act.php?id=BOE-A-2007-6115 [19 de abril de 2019].

Liang, Bin y Hu, Ming (2018), "A typology of sex offending against minors: an empirical study of rape and molestation cases in China", en Sexual Abuse, vol. 30, núm. 8. DOI: 10.1177/1079063217715017. Disponible en:

https://journals.sagepub.com/doi/full/10.1177/1079063217715017 [13 de mayo de 2019]. 
Logan, T. K. et al. (2015), "Silenced suffering: The need for a better understanding of partner sexual violence", en Trauma, Violence \& Abuse, vol. 16, núm. 2. DOI: 10.1177/1524838013517560. Disponible en: https://journals.sagepub.com/doi/ full/10.1177/1524838013517560 [5 de abril de 2019].

López, Silvia et al. (2017), "Child and adolescent sexual abuse in women seeking help for sexual and reproductive mental health problems: prevalence, characteristics, and disclosure", en Journal of child sexual abuse, vol. 26, núm. 3. Disponible en: https:// www.tandfonline.com/doi/abs/10.1080/10538712.2017.1288186 [9 de mayo de 2019].

Marcos, Liliana et al. (2017), "Ojos que no quieren ver. Los abusos sexuales a niños y niñas en España y los fallos del sistema”, en Save the Children España. Disponible en: https:// www.savethechildren.es/sites/default/files/imce/docs/ojos_que_no_quieren_ ver_12092017_web.pdf [9 de mayo de 2019].

Mendes, Kaitlynn et al. (2018), "\#MeToo and the promise and pitfalls of challenging rape culture through digital feminist activism”, en European Journal of Women's Studies, vol. 25, núm. 2. Disponible en: https://scholar.google.es/scholar?hl=es\&as_sdt=0\%2C5 $\& \mathrm{q}=10.1177 \% 2 \mathrm{~F} 1350506818765318 \& \mathrm{btnG}=[3$ de junio de 2019].

Morales, José Francisco et al. (2016), "Gender and Bullying: application of a three factor model of gender stereotyping”, en Sex Roles, vol. 74. Disponible en: https://link.springer.com/article/10.1007/s11199-015-0463-3 [6 de junio de 2019].

Morrison-Beedy, Dianne y Grove, Linsey (2018), "Adolescent girls' experiences with sexual pressure, coercion, and victimization: \#MeToo", en Worldviews on Evidence-Based Nursing, vol. 15, núm. 3. Disponible en: https://sigmapubs.onlinelibrary.wiley.com/ doi/full/10.1111/wvn.12293 [6 de junio de 2019].

OMS (2005), Estudio a fondo sobre todas las formas de violencia contra la mujer, Estados Unidos: Naciones Unidas. Disponible en: https://www.cepal.org/mujer/noticias/ paginas/1/27401/InformeSecreGeneral.pdf [6 de junio de 2019].

Ortega, Rosario et al. (2010), "Peer sexual harassment in adolescent girls: A cross-national study (Spain-Italy)", en International Journal of Clinical and Health Psychology, vol. 10, núm. 2. Disponible en: https://www.redalyc.org/pdf/337/33712250003.pdf [6 de junio de 2019].

Park, Andrea (2017), "MeToo reaches 85 countries with 1.7M tweets", en CBS News, vol. 24. Disponible en: https://www.cbsnews.com/news/metoo-reaches-85-countries-with1-7-million-tweets/ [3 de junio de 2019].

Patrick, Julie (2018), Ending sexual violence in one generation: A progress report for the United States 2018, Estados Unidos: Raliance.

Pereda, Noemí et al. (2018), “¿Qué factores inciden para romper el silencio de las víctimas de abuso sexual?”, en Revista Española de Investigación Criminológica, vol. 16. Disponible en: https://reic.criminologia.net/index.php/journal/article/view/195 [13 de mayo de 2019].

Pernas, Begoña et al. (2000), La dignidad quebrada. Las raices del acoso sexual en el trabajo, España: Los Libros de la Catarata.

Quinn, Beth (2002), "Sexual harassment and masculinity: The power and meaning of "girl watching", en Gender y Society, vol. 16, núm. 3. DOI: 10.1177/0891243202016003007. Disponible en: https://journals.sagepub.com/doi/ abs/10.1177/0891243202016003007 [9 de mayo de 2019]. 
Reed, Shon et al. (2019), "Friends, family, and boyfriends: An analysis of relationship pathways into commercial sexual exploitation", en Child abuse y neglect, vol. 90. Disponible en:

https://www.sciencedirect.com/science/article/pii/S0145213419300365 [6 de junio de 2019].

Rodríguez-Martínez, Pilar y Cuenca-Piqueras, Cristina (2019), "Interactions between direct and structural violence in sexual harassment against Spanish and unauthorized migrant women”, en Archives of sexual behavior, vol. 48, núm. 2. Disponible en: https:// link.springer.com/article/10.1007/s10508-018-1265-9 [6 de julio de 2020].

Rodríguez, Yolanda et al. (2018), "Del sexting al cibercontrol en las relaciones de pareja de adolescentes españoles: análisis de sus argumentos", en Revista Latinoamericana de Psicología, vol. 50, núm. 38. DOI: 10.14349/rlp. 2018.v50.n3.4 Disponible en:

http://www.scielo.org.co/scielo.php?script=sci_arttext\&pid =S0120-05342018000300170 [6 de junio de 2019].

Rodríguez, Yolanda et al. (2019), "Una radiografía del acoso sexual en España”, en Blanco Martín, Agustín [ed.], Informe España 2019, España: Cátedra José María Patino de la Cultura del Encuentro.

Rodríguez, Yolanda et al. (2014), "Programas de prevención de abusos sexuales a menores (PPASM): el papel de la educación sexual”, en Lameiras Fernández, María y Orts Berenguer, Enrique [eds.], Delitos sexuales contra menores, abordaje psicológico, jurídico y policial, España: Tirant lo Blanch.

Schuster, Isabel y Krahé, Barbara (2019), "Prevalence of sexual aggression victimization and perpetration in Chile: A systematic review”, en Trauma, Violence \& Abuse, vol. 20, núm. 2. DOI: 10.1177/1524838017697307. Disponible en: https://journals. sagepub.com/doi/full/10.1177/1524838017697307 [10 de junio de 2019].

Smith, Sharon et al. (2018), The National Intimate Partner and Sexual Violence Survey (NISVS): 2015 Data Brief-Updated Release, Estados Unidos: National Center for Injury Prevention and Control, Centers for Disease Control and Prevention. Disponible en: https://www.cdc.gov/violenceprevention/pdf/2015data-brief508.pdf [8 de julio de 2020].

Tuerkheimer, Deborah (1997), "Street harassment as sexual subordination: The phenomenology of gender-specific harm”, en Wisconsin Women's Law Journal, vol. 12, núm. 2. Disponible en: https://heinonline.org/HOL/LandingPage?handle=hein. journals $/$ wiswo $12 \&$ div $=11 \&$ id $=\&$ page $=[19$ de abril de 2019] .

Vega-Gea, Esther et al. (2016), "Peer sexual harassment in adolescence: Dimensions of the sexual harassment survey in boys and girls", en International Journal of Clinical and Health Psychology, vol. 16, núm. 1. DOI: 10.1016/j.ijchp.2015.08.002. Disponible en: https://idus.us.es/ handle/11441/52391; jsessionid=25FB99EB61B77D8A53F49C7AAAB2FDDC? [19 de abril de 2019].

Witt, Andreas et al. (2018), “The prevalence of sexual abuse in institutions: Results from a representative population-based sample in Germany”, en Sexual Abuse, vol. 31, núm. 6. DOI: $10.1177 / 1079063218759323$. Disponible en: https://journals.sagepub.com/ doi/full/10.1177/1079063218759323 [5 de abril de 2019]. 
Yolanda Rodríguez Castro. Profesora contratada doctora, Facultad de Educación y Trabajo Social, Universidad de Vigo (España). Líneas de investigación: sexismo, estereotipos de género, (ciber)violencia de género, sexting y educación sexual. Publicaciones recientes: Rodríguez Castro, Yolanda et al. (2017), "Spanish adolescents' attitudes towards sexting: validation of a scale", en Computers in Human Behavior, vol. 73, disponible en: http://dx.doi. org/10.1016/j.chb.2017.03. Rodríguez Castro, Yolanda et al. (2018), "Del sexting al cibercontrol en las relaciones de pareja de adolescentes españoles: análisis de sus argumentos", en Revista Latinoamericana de Psicología, vol. 50, núm. 3, disponible en: http://dx.doi.org/10.14349/rlp.2018.v50.n3.4 . Adá Lameiras, Alba y Rodríguez Castro, Yolanda (2020), "The presence of female athletes and non-athletes on sports media Twitter", en Feminist Media Studies, disponible en: https://doi.org/10.1080/14680777.2020.1732439.

Rosana Martínez Román. Graduada en Educación Social por la Universidad de Vigo. Becaria Predoctoral en la Facultad de Educación y Trabajo Social, Universidad de Vigo (España). Líneas de investigación: acoso sexual, acoso por razón de sexo, abuso sexual y agresión sexual. Publicaciones recientes: Alonso, Patricia et al. (2018), "El sexting a través del discurso de adolescentes españoles", en Saúde e Sociedade, vol. 27, núm. 2, disponible en: https://doi.org/10.1590/S0104-12902018171835. Martínez Román, Rosana y Rodríguez Castro, Yolanda (2017), "Análisis de los discursos de las profesionales de un centro de atención a víctimas de trata con fines de explotación sexual", en Revista de Estudios e Investigación en Psicologia y Educación, vol. 5, disponible en: https://doi.org/10.17979/reipe.2017.0.05.2384. Martínez Román, Rosana et al. (2017), "Análisis del acoso sexual y por razón de sexo desde la perspectiva de víctima”, en Revista de Estudios e Investigación en Psicología y Educación, vol. 2, disponible en: https://doi.org/10.17979/ reipe.2017.0.2.2383.

Patricia Alonso Ruido. Profesora contratada interina en la Facultad de Ciencias de la Educación de Santiago de Compostela (España). Líneas de investigación: sexting, redes sociales, (ciber)violencia de género y educación sexual. Publicaciones recientes: Alonso, Patricia et al. (2018), "El sexting a través del discurso de adolescentes españoles", en Saúde e Sociedade, vol. 27, núm. 2, disponible en: https://doi.org/10.1590/S0104-12902018171835. Rodríguez Castro, Yolanda et al. (2018), "Del sexting al cibercontrol en las relaciones de pareja de adolescentes españoles: análisis de sus argumentos”, en Revista Latinoamericana de Psicología, vol. 50, núm. 3, disponible en: 
http://dx.doi.org/10.14349/rlp.2018.v50.n3.4 . Rodríguez Castro, Yolanda et al. (2017), "Spanish adolescents' attitudes towards sexting: validation of a scale", en Computers in Human Behavior, vol. 73, disponible en: http:// dx.doi.org/10.1016/j.chb.2017.03.

María Victoria Carrera Fernández. Profesora ayudante doctora, Facultad de Educación y Trabajo Social, Universidad de Vigo (España). Líneas de investigación: diversidad sexual, teoría Queer, homofobia, lesbofobia y educación sexual. Publicaciones recientes: Carrera Fernández, María Victoria et al. (2019), "Gender-bashing in adolescents: structural relations with heterosexual matrix, racism/xenophobia and attitudes toward bullying", en Journal of School Health, disponible en: http://dx.doi.org/10.1111/ josh.12778. Carrera Fernández, María Victoria et al. (2019), "Patrolling the boundaries of gender: beliefs, attitudes and behaviors towards trans and gender diverse people in Portuguese adolescents", en International Journal of Sexual Health, disponible en: https://doi.org/10.1080/19317611.2019.17 01170. Carrera Fernández, María Victoria et al. (2018), "Performing intelligible genders through violence: bullying as gender practice and heteronormative control", en Gender and Education, vol. 30, núm. 3, disponible en: https://doi.org/10.1016/10.1080/09540253.2016.1203884. 